\title{
Circular RNA-DPP4 serves an oncogenic role in prostate cancer progression through regulating miR-195/cyclin D1 axis
}

Deping Yang ${ }^{1 \dagger}$, Bo Yang ${ }^{2 \dagger}$, Yanjun Zhu ${ }^{3 \dagger}$, Qianlin Xia ${ }^{4}$, Yan Zhang ${ }^{1}$, Xin Zhu ${ }^{5}$, Jianming Guo ${ }^{3}$, Tao Ding ${ }^{5^{*}}$ and Jianghua Zheng ${ }^{1 *}$ (D)

\begin{abstract}
Background: Recently, more and more studies have highlighted the critical regulatory roles of circular RNAs (circRNAs), a class of non-coding RNAs, in the progression of many human cancers, including prostate cancer (PCa). circRNA microarray analysis was performed to identify circRNAs that are differentially expressed in PCa tissues.

Methods: 104 pairs of PCa tissues and matched adjacent normal prostate tissues (at least $2 \mathrm{~cm}$ distal to the tumor margin) were obtained. circRNA microarray analysis was performed on four pairs of PCa tissues and matched adjacent normal prostate tissues to investigate the potential involvement of circRNAs in PCa. Flow cytometric analysis was performed to investigate whether the effect of circDPP4 on PCa cell proliferation was associated with the alteration in cell cycle progression. The role of circDPP4 in PCa tumor growth was further explored in vivo.

Results: We found that circDPP4 was overexpressed in PCa tissues and cell lines, and its expression was closely associated with Gleason score and clinical stage of PCa patients. In vitro loss- and gain-of-function experiments demonstrated that circDPP4 knockdown inhibited, whereas circDPP4 overexpression promoted the proliferation, migration, invasion and cell cycle progression of PCa cells. Knockdown of circDPP4 also suppressed PCa tumor growth in vivo. We further found that circDPP4 functioned as a competing endogenous RNA (ceRNA) for miR-195 in PCa cells, and miR-195 negatively regulated the expression of oncogenic cyclin D1. Rescue experiments suggested that restoration of miR-195 blocked the oncogenic role of circDPP4 in PCa cells.
\end{abstract}

Conclusions: Taken together, our findings revealed a novel regulatory mechanism between circDPP4 and miR-195/ cyclin D1 axis, and offered novel strategies for the treatment of PCa.

Keywords: Prostate cancer, Circular RNA DPP4 (circDPP4), microRNA-195, Cell cycle, Cyclin D1

\section{Background}

Globally, prostate cancer (PCa) is the second most frequent cancer and the fifth leading cause of cancer-related

\footnotetext{
*Correspondence: dingtao1006@126.com; ZHENGjh2016@vip.163.com ${ }^{+}$Deping Yang, Bo Yang and Yanjun Zhu contributed equally to the work 1 Department of Laboratory Medicine, Shanghai University of Medicine \& Health Sciences Affiliated Zhoupu Hospital, Shanghai 201318, China ${ }^{5}$ Department of Urology, The Sixth People's Hospital South Campus, Shanghai Jiao Tong University, Shanghai 201489, China

Full list of author information is available at the end of the article
}

deaths in male [1]. Over the last few years, the incidence and mortality rates of $\mathrm{PCa}$ have increased rapidly in China, including Shanghai $[2,3]$. Despite the fact that great progress has been made in the diagnostic and therapeutic methods, the management of PCa still remains one of the most serious medical challenges. Accordingly, getting a better understanding of the potential carcinogenetic mechanisms is of critical importance to identify novel diagnostic and therapeutic targets for PCa patients. original author(s) and the source, provide a link to the Creative Commons licence, and indicate if changes were made. The images or other third party material in this article are included in the article's Creative Commons licence, unless indicated otherwise in a credit line to the material. If material is not included in the article's Creative Commons licence and your intended use is not permitted by statutory regulation or exceeds the permitted use, you will need to obtain permission directly from the copyright holder. To view a copy of this licence, visit http://creativecommons.org/licenses/by/4.0/. The Creative Commons Public Domain Dedication waiver (http://creativeco mmons.org/publicdomain/zero/1.0/) applies to the data made available in this article, unless otherwise stated in a credit line to the data. 
Circular RNAs (circRNAs), a large group of noncoding RNAs, are characterized by their covalently closed loop structures without a $5^{\prime}$ cap or a $3^{\prime}$ poly A tail [4]. circRNAs are abundant in the human transcriptome, and compared with linear RNAs, circRNAs are more stable and conserved in sequence. The term "circRNA" was first proposed by Sanger in 1976 [5], and in recent years, emerging evidence has shown the biological roles of circRNAs in multiple processes such as cell growth, migration and invasion, which are thought to be critical for the tumorigenesis and progression of various human cancers [6, 7]. Mechanically, circRNAs function as microRNA (miRNA) sponges, RNA-binding proteins and transcriptional regulators, involving the pathogenesis of these malignancies $[8,9]$. Many studies demonstrated that circRNAs is mainly involved in the regulation of gene expression and specific biological functions by sponging miRNAs. For instance, circRNA CDR1 was reported as a sponge of miR-432-5p to regulate expression of E2F3, a direct target gene of miR-432-5p, and promote proliferation, migration and invasion of PCa cells [10]. circANKS1B plays an oncogenic role in the progression of $\mathrm{PCa}$ via sponging miR-152-3p and upregulating TGF- $\alpha$ expression [11].

As an important regulator of cell cycle, cyclin D1 is involved in the transition from $\mathrm{G} 1$ to $\mathrm{S}$ phase by regulating cyclin-dependent kinase (CDK) 4 and CDK6 [12]. Dysregulation of cyclin D1 expression drives uncontrolled cell proliferation and results in the development of human cancers. It has been reported that the expression level of cyclin D1 was markedly upregulated in human PCa, breast cancer, non-small cell lung cancer and hepatocellular carcinoma [13-16]. Several studies have shown that cyclin D1 expression could be negatively regulated by miR-195 to affect the tumorigenicity and invasiveness of laryngeal squamous cell carcinoma and glioblastoma cells [17, 18]. Recently, Duan et al. reported the biological roles of circMYLK/ miR-195/cyclin D1 axis in the development and progression of laryngeal squamous cell carcinoma, providing a novel therapeutic target for these patients [17]. However, the relationship between circRNAs and miR$195 /$ cyclin D1 axis in the pathogenesis of PCa remains unclear.

In the present study, we identified a novel circRNA circDPP4, which is significantly upregulated in PCa tissues, by human circRNA microarray analysis. Subsequently, we performed a series of functional assays and molecular mechanism analysis to investigate the effect of its overexpression on biological characteristics of PCa cells and determine whether circDPP4 functions as an oncogene to promote the development and progression of $\mathrm{PCa}$ via sponging miR-195 and inducing cyclin D1 overexpression.

\section{Methods \\ Clinical specimens}

104 pairs of PCa tissues and matched adjacent normal prostate tissues (at least $2 \mathrm{~cm}$ distal to the tumor margin) were obtained from patients who were diagnosed with PCa by histopathology and undergone surgery at Shanghai University of Medicine \& Health Sciences Affiliated Zhoupu Hospital. In this study, early stage PCa was defined as a tumor with $\leq$ stage cT2a-T2c without the evidence of metastases; otherwise, advanced stage PCa was defined. The clinicopathological data of these patients are listed in Additional file 4: Table S1. All patients did not receive any other treatment prior to operation. After removal from the body, tissue samples were snap-frozen in liquid nitrogen and stored at $-80^{\circ} \mathrm{C}$. The use of human tissues was approved by the Ethics Committee of Shanghai University of Medicine \& Health Sciences Affiliated Zhoupu Hospital, and all patients or their relatives signed the informed consent.

\section{CircRNA microarray analysis}

A total of 4 pair matched $\mathrm{PCa}$ and adjacent normal tissues were used for microarray analysis to identify differentially expressed circRNAs involved in tumorigenesis and progression of $\mathrm{PCa}$ as previously described [19]. Total RNA was extracted using TRIzol reagent (Invitrogen, Carlsbad, CA, USA). RNA concentration and quality were analyzed using Agilent Bioanalyzer 2100 (Agilent Technologies, Santa Clara, CA, USA). Then, RNA samples were amplified, labeled using Low Input Quick Amp WT Labeling Kit (Agilent Technologies), and hybridized onto SBC-ceRNA (4*180 K) (BH170234; Shanghai Bohao Biotechnology co., LTD, Shanghai, China). The hybridized microarrays were washed and then scanned with Agilent Microarray Scanner (Agilent Technologies). Quantile normalization and subsequent data processing were performed using the $\mathrm{R}$ software limma package. $\mid \log _{2}$ fold change $\mid>1.0$ and $P$ value $<0.05$ were considered significant. Hierarchical clustering analysis was performed to show the differentially expressed circRNAs among samples.

\section{Cell culture and transfection}

Four human PCa cell lines (PC3, DU145, LNCaP and 22RV1), a human prostate epithelial cell line (RWPE1) and human embryonic kidney cell line (HEK293T) purchased from American Type Culture Collection (Manassas, VA, USA) were cultured in RPMI 1640 medium (Invitrogen) containing 10\% fetal bovine serum (FBS; 
Biowest, Loire, France), $100 \mathrm{U} / \mathrm{ml}$ penicillin sodium and $100 \mathrm{mg} / \mathrm{ml}$ streptomycin sulfate at $37{ }^{\circ} \mathrm{C}$ in an atmosphere containing $5 \% \mathrm{CO}_{2}$. The cells were periodically confirmed negative for mycoplasma contamination and were authenticated by DNA profiling.

MiR-195 mimics, miR-195 inhibitor, mimics control and inhibitor control were chemically synthesized by GenePharma Co. Ltd. (Shanghai, China). The synthesized circDPP4 gene fragment was inserted into the pcDNA3.1 vector (Invitrogen) to construct overexpression vector. An empty pcDNA3.1 vector was used as a negative control. Short-hairpin RNA oligo 5'-UGUUCUUCUUGU UUGACAGGA-3') directed against circDPP4 was synthesized and inserted into the SuperSilencing shRNA plasmid pGPU6/Neo (GenePharma Co. Ltd.). Cells at 70-80\% confluence were used for transfection using Lipofectamine 2000 (Invitrogen).

\section{RNA extraction and RT-qPCR analysis}

The isolated RNA was reverse transcribed into cDNA by using the PrimeScript ${ }^{\mathrm{TM}}$ RT reagent kit (TaKaRa, Dalian, China). qPCR reactions were then carried out on an ABI PRISM 7500 fast Sequence Detection System (Applied Biosystems, Foster City, CA, USA) using the SYBR Premix Ex Taq II kit (TaKaRa). The $2^{-\Delta \Delta C t}$ method was used to determine relative expression levels of crircDPP4 and miR-195 in PCa tissues and cell lines [20]. GAPDH was chosen as the internal control for circRNA and mRNA. U6 was chosen as the internal control for miRNA. The primer sequences of circDPP4 and GAPDH are crircDPP4 forward: 5'-AATGAGAGGGAAGAGCGG AG-3'; crircDPP4 reverse: 5'-ACATCCACGTCCTTT CCCAT-3'; GAPDH forward: 5'-CACATCGCTC AGA CACCATG-3'; GAPDH reverse: 5'-TGACGGTGCCAT GGAA TTTG-3'. Other primer sequences for RT-qPCR assay are presented in Additional file 4: Table S1.

\section{CCK-8 assay}

Cell proliferation was detected using Cell Counting Kit-8 (CCK-8; Dojindo Laboratories, Kumamoto, Japan). Cells were seeded at a density of 2000 cells/well in 96-well plates. $10 \mu \mathrm{l}$ of CCK-8 solution was added into each well, and the plants were incubated for another $2 \mathrm{~h}$. The absorbance was measured with a microplate reader (Dynex, Chantilly, VA, USA).

\section{EdU staining assay}

Cells were cultured in 24-well plates, and $10 \mu \mathrm{M}$ of EdU reagent (RiboBio, Guangzhou, China) was added to each well according to the manufacturer's protocol. After $2 \mathrm{~h}$ of incubation at $37^{\circ} \mathrm{C}$, the cells were fixed with $4 \%$ paraformaldehyde for $30 \mathrm{~min}$, and then the nuclei were stained with Hoechst 33,342 for 5 min. Cells were visualized under a fluorescent microscope (Olympus, Tokyo, Japan) and the ratio of EdU-positive cells to total cells was calculated.

\section{Transwell assay}

$2 \times 10^{5}$ cells were resuspended in $100 \mu \mathrm{l}$ of medium and seeded into the upper chambers of Transwell plates (8- $\mu \mathrm{m}$ pore size; Corning-Costar, Cambridge, MA, USA) coated with or without Matrigel (Corning-Costar) and containing $600 \mu \mathrm{l}$ of medium supplemented with $10 \%$ FBS in the lower chambers. Following $24 \mathrm{~h}$ of incubation, the cells on the upper membrane surface were carefully scraped off with cotton swabs, and the cells on the lower membrane surface were fixed with $4 \%$ paraformaldehyde, stained with $0.1 \%$ crystal violet and photographed under a light microscope. Five random fields were counted per chamber.

\section{Cell cycle analysis}

The Cycle TEST PLUS DNA Reagent Kit (BD Biosciences, San Jose, CA, USA) was used to detect cell cycle progression. The cells were collected and fixed in $75 \%$ ethanol overnight at $4{ }^{\circ} \mathrm{C}$. Then the fixed cells were incubated with $100 \mu \mathrm{l}$ RNase A reagent (Keygen Biotech, Nanjing, China) for $30 \mathrm{~min}$ at $37^{\circ} \mathrm{C}$, and stained with $20 \mu \mathrm{g} / \mathrm{ml}$ PI for $10 \mathrm{~min}$ at room temperature. The percentage of cells in G0/G1, S, and G2/M phases was analyzed by flow cytometry (BD Biosciences).

\section{Western blot analysis}

Total protein was extract from tissues and cells using RIPA lysis buffer (Beyotime, Shanghai, China) and quantified by a BCA Protein Assay Kit (Solarbio, Beijing, China). Equivalent amount of proteins were separated by SDS-polyacrylamide gel electrophoresis and transferred onto PVDF membranes (Millipore, Billerica, MA, USA). The membranes were blocked with $5 \%$ skimmed milk and probed with specific primary antibodies against cyclin D1 (1:500; Proteintech, Chicago, IL, USA) and GAPDH (1:1000; Cell Signaling Technology, Danvers, MA, USA), followed by incubation with appropriate HRP-linked secondary antibodies (1:1000; Beyotime) for $1 \mathrm{~h}$ at room temperature. The bands were visualized using an enhanced chemiluminescence kit (Santa Cruz, Dallas, TX, USA). To ensure equal protein loading, GAPDH was considered as the loading control.

\section{Subcellular fractionation location}

The PARIS Kit (Invitrogen) was used to separate the nuclear and cytoplasmic fractions of PCa cells. RT-qPCR 
analysis was performed to detect the expression levels of circDPP4, GAPDH and U6 in the nuclear and cytoplasmic fractions. GAPDH was used as the cytoplasmic control, and U6 was used as the nuclear control.

\section{Fluorescence in situ hybridization (FISH) assay}

The localization of circDPP4 and miR-195 in PCa cells was detected by fluorescence in situ hybridization (FISH) kit (ThermoFishers, USA). PC3 cells were harvested and fixed with $4 \%$ paraformaldehyde (PFA) for $15 \mathrm{~min}$ at room temperature. The fluorescein-labeled probes specific for circDPP4 (green fluorescence) and miR-195 (red fluorescence) were hybridized at $37{ }^{\circ} \mathrm{C}$ overnight. Moreover, cell nuclei (blue fluorescence) were stained with 4',6-diamidino-2-phenylindole (DAPI). All procedures were performed according to the manufacturer's ViewRNA ISH Assays protocol. A confocal microscopy was used to observe the presence of circDPP4 and miR-195 in PCa cells.

\section{Dual-luciferase reporter assay}

The fragment from circDPP4 or cyclin D1 mRNA 3'-UTR containing the predicted miR-195 binding site was amplified by PCR and cloned into psiCHECK-2 luciferase reporter vector (Promega, Madison, WI, USA). circDPP4-MUT and cyclin D1-MUT reporters were generated using Quickchange XL site-directed mutagenesis kit (Agilent Technologies). All constructs were confirmed by DNA sequencing. PC 3 cells at $80 \%$ confluence in 96-well plate were co-transfected with the luciferase reporter vectors and miR-195 mimics or mimics control using Lipofectamine 2000. After 48 h of transfection, luciferase activity was measured using the Dual-Luciferase Reporter Assay system (Promega).

\section{RNA-pull down assay}

The biotin-labeled probe with wild type miR-195 (biotin-wt-miR-195) and mutant type miR-195 (biotinmut-miR-195) and negative control (biotin-NC) were synthesized. Then, they were transfected into PC3 and LNCaP cells. After $48 \mathrm{~h}$, the cells were harvested, lysed by lysis buffer and incubated with streptavidin beads for $2 \mathrm{~h}$ at $4{ }^{\circ} \mathrm{C}$. Next, the beads were washed by lysis buffer for three times, and the RNA complex was purified with TRIzol reagent. qRT-PCR analysis was conducted to detect the abundance of circDPP4 in PCa cells.

\section{Animal tumor xenograft model}

Twelve male nude mice (athymic, Balb/c nu/nu, aged 5-6 weeks, weighting 18-20 g) were purchased from the Slac Laboratory Animal Center (Shanghai, China) and kept under specific pathogen-free conditions. $1 \times 10^{6}$
PC3 cells stably transfected with sh-circDPP4 or sh-NC were resuspended in $200 \mu \mathrm{l}$ of PBS and inoculated subcutaneously into a single side of the posterior flank of nude mice. Six mice were included in each group. Tumors were measured using a caliper every week and tumor volume was calculated following the formula: $V=0.5 \times D \times d^{2}$ (V, volume; $\mathrm{D}$, longitudinal diameter; $d$, latitudinal diameter). Five weeks after cell inoculation, mice were sacrificed by $\mathrm{CO}_{2}$ asphyxiation, and the xenograft tumors were excised, weighted and subjected to $\mathrm{H} \& \mathrm{E}$ staining and immunohistochemistry (IHC) analysis of Ki-67 protein.

All experimental procedures involving the use of animals were approved by the Ethics Committee of Zhongshan Hospital, and all efforts were made to minimize animal suffering, according to the NIH Guide for the Care and Use of Laboratory Animals.

\section{Statistical analysis}

All data were analyzed by GraphPad Prism 6.0 software (GraphPad Software, San Diego, CA, USA) and SPSS 16.0 software (SPSS, Chicago, IL, USA). Comparisons between groups were analyzed using the Student's $t$-test and one-way analysis of variance or $\chi^{2}$ test, as appropriate. Correlation between circDPP4 and miR-195 expression in PCa tissues was evaluated by Pearson's correlation analysis. $P<0.05$ was considered to indicate a statistically significant difference. Original images were included in Additional file 6.

\section{Results \\ CircDPP4 is upregulated in human PCa tissues and significantly correlated with the disease progression} First, circRNA microarray analysis was performed in four pairs of PCa tissues and matched adjacent normal prostate tissues to investigate the potential involvement of circRNAs in PCa. The relevant findings were previously described [19]. Based on the threshold of $\mid \log 2$ fold change $\mid>2.0$ and adjusted $\mathrm{P}<0.05$, a total of 117 circRNAs were significantly upregulated, and 904 circRNAs were downregulated in four PCa tissue samples (Additional file 1: Fig. S1 and Additional file 6).

We found that hsa_circ_0056881, derived from gene DPP4, was among the top upregulated circRNAs. Hsa circ_0056881 was therefore termed as "circDPP4". We then validated the expression of circDPP4 in a cohort of 104 pair of $\mathrm{PCa}$ tissues and matched adjacent normal tissues using RT-qPCR analysis. As shown in Fig. 1A, the expression of circDPP4 was remarkably increased in PCa tissues than in the matched adjacent normal tissues. Moreover, expression of circDPP4 increased with the severity of disease as it is higher in stage cT3a-T4 PCa than in stage $\mathrm{cT} 2 \mathrm{a}-\mathrm{T} 2 \mathrm{c}($ Fig. $1 \mathrm{~B}, \mathrm{P}<0.05)$. In addition, 

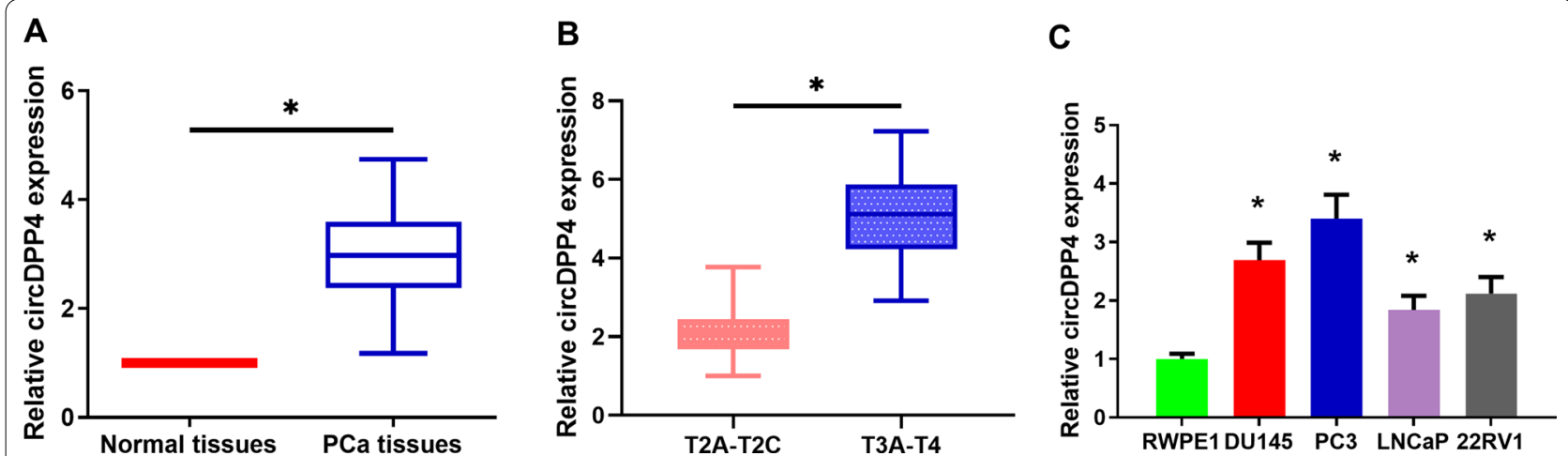

Fig. 1 circDPP4 is upregulated in human PCa tissues. A RT-qPCR analysis of circDPP4 expression levels in 104 pairs of PCa tissues and adjacent normal tissues. ${ }^{*} P<0.05$ vs. normal tissues. B Expression of circDPP4, showing higher level in late stage than in early stage of PCa patients. $\mathbf{C}$ RT-qPCR analysis of circDPP4 expression levels in PCa cell lines and normal counterpart. ${ }^{*} P<0.05$ vs. normal cells

circDPP4 showed a higher expression in four PCa cell lines (PC3, DU145, LNCaP and 22RV1) compared to normal prostate epithelial cell line (RWPE1) (Fig. 1C).

To further evaluate the clinical significance of circDPP4 in $\mathrm{PCa}$, we explored the correlation between circDPP4 expression and clinicopathological features of $\mathrm{PCa}$ patients. The $104 \mathrm{PCa}$ patients were allocated into a high expression group ( $\mathrm{n}=52$; $>$ median) and a low expression group ( $\mathrm{n}=52$; $<$ median) based on the median expression level of circDPP4. As indicated in Additional file 5: Table S2, PCa patients with high circDPP4 expression had a higher Gleason score $(\mathrm{P}=0.015)$ and were at more advanced clinical stage $(\mathrm{P}=0.026)$ than those with low circDPP4 expression.

\section{CircDPP4 promotes PCa cell proliferation, migration and invasion in vitro}

The increased circDPP4 expression in human PCa samples prompted us to investigate the biological role of circDPP4 in PCa tumorigenesis. circDPP4 was knocked down in PC3 cells by transfection of sh-circDPP4 and was overexpressed in LNCaP cells by transfection of pcDNA3.1-circDPP4. The transfection efficacies were confirmed by RT-qPCR analysis (Fig. 2A). The results of CCK-8 assay showed that proliferation of sh-circDPP4transfected PC3 cells was notably suppressed, whereas overexpression of circDPP4 enhanced proliferation of LNCaP cells (Fig. 2B). In addition, as indicated by EdU staining assay, circDPP4 knockdown reduced whereas its overexpression increased the proportion of EdU incorporated PCa cells (Fig. 2C). We further evaluated the role of circDPP4 in migration and invasion of PCa cells. The results of Transwell assay revealed that migratory and invasive capacities of PCa cells were significantly weakened by circDPP4 knockdown and remarkably enhanced by circDPP4 overexpression (Fig. 2D).

\section{CircDPP4 promotes PCa cell cycle progression}

Moreover, flow cytometric analysis was performed to investigate whether the effect of circDPP4 on PCa cell proliferation was associated with the alteration in cell cycle progression. The results indicated that circDPP4 overexpression promoted cell cycle progression of LNCaP cells, whereas the proportion of sh-circDPP4transfected PC3 cells at G0/G1 phase was notably increased (Fig. 3A). The effects of circDPP4 on cell cyclerelated proteins in $\mathrm{PCa}$ cells were further evaluated. As shown in Fig. 3B, cyclin D1 was the only up-regulated protein in LNCaP cells with circDPP4 overexpression. On the contrary, circDPP4 knockdown reduced cyclin D1 protein expression in PC3 cells.

\section{Knockdown of circDPP4 inhibits PCa tumor growth in vivo} The role of circDPP4 in PCa tumor growth was further explored in vivo. In accordance with the in vitro findings, knockdown of circDPP4 led to a remarkable attenuation of PCa tumor growth (Fig. 4A). At 5 weeks of post-injection, the average tumor weight of the shcircDPP41 group was obviously reduced (Fig. 4B), and the downregulation of circDPP4 in the tumors formed from sh-circDPP4-transfected cells was validated by RT-qPCR analysis (Fig. 4C). Moreover, as shown in Fig. 4D, cyclin D1 protein expression was reduced in the tumor tissues from the sh-circDPP4 group. The staining intensity of proliferation antigen $\mathrm{Ki}-67$ in the tumor tissues was also notably reduced by circDPP4 knockdown (Fig. 4E).

\section{CircDPP4 acts as a ceRNA for miR-195 in PCa cells}

We further uncovered that circDPP4 was predominantly localized in the cytoplasmic fraction of PC3 and $\mathrm{LNCaP}$ cells (Fig. 5A), indicating that circDPP4 might function 
(See figure on next page.)

Fig. 2 circDPP4 promotes PCa cell proliferation, migration and invasion in vitro. A RT-qPCR analysis of circDPP4 expression levels in PC3 and LNCaP cells after transfection. $\mathbf{B}$ The proliferation of PC3 and LNCaP cells after transfection detected by CCK-8 assay. C The proliferation of PC3 and LNCaP cells after transfection detected by EdU staining assay. D The migration and invasion of PC3 and LNCaP cells after transfection detected by transwell assay. ${ }^{*} P<0.05$ vs. sh-NC or empty vector-transfected cells

as a ceRNA to sequester miRNAs in PCa cells. Through the Starbase database (http://starbase.sysu.edu.cn/index. php), we found that circDPP4 has a binding site for miR195 (Fig. 5B). To validate the direct binding relationship between circDPP4 and miR-195, dual-luciferase reporter assay was conducted, and the results showed that the luciferase activity was reduced by more than $40 \%$ when PC3 cells were co-transfection with circDPP4WT reporter and miR-195 mimics (Fig. 5C). Moreover, RNA pull-down assay revealed that circDPP4 was significantly enriched with biotin-labeled wt-miR-195 in PCa cells compared with negative control and/or biotinlabeled mut-miR-195. These findings further supported a direct interaction between circDPP4 and miR-195 in PCa cells (Fig. 5D). We also found that miR-195 expression was decreased in circDPP4 overexpressed LNCaP cells, but increased in circDPP4 knockdown PC3 cells (Fig. 5E). Besides, expression of miR-195 was significantly decreased in PCa tissues and cell lines (Fig. 5F, G). MiR195 expression was inversely correlated with circDPP4 expression in $\mathrm{PCa}$ tissues of 20 randomly selected patients (Fig. 5H).

\section{Cyclin D1 is a downstream target of miR-195 in PCa cells}

Bioinformatics analysis also demonstrated that there are two conserved binding sites for miR-195 on the 3'-UTR of cyclin D1 mRNA (Additional file 2: Fig. S2A). In addition, as shown in Additional file 2: Fig. S2B, miR-195 mimics significantly inhibited luciferase activity of cyclin D1-WT reporter but not the MUT reporter in HEK293T cells. Meanwhile, cyclin D1 protein level was decreased by miR-195 mimics in PC3 cells and increased by miR195 inhibitor in LNCaP cells (Additional file 2: Fig. S2C). Furthermore, we found that miR-195 overexpression suppressed whereas miR-195 inhibition promoted the proliferation of PCa cells (Additional file 2: Fig. S2D).

\section{MiR-195 blocks the oncogenic role of circDPP4 in PCa cells}

We then performed the rescue experiments and demonstrated that restoration of miR-195 blocked the effects of circDPP4 overexpression on the proliferation and cell cycle distribution of LNCaP cells (Fig. 6A, B). In contrast, co-transfection with miR-195 inhibitor abolished the effects of circDPP4 knockdown on the proliferation and cell cycle distribution of PC3 cells. Furthermore, we performed RNA-ISH assay to validate the localization of circDPP4 and miR-195 in tumor tissue and PC3 cell line and found that circDPP4 and miR-195 are co-localized (Additional file 3: Fig. S3).

\section{Discussion}

The role of non-coding RNAs including circRNAs has attracted extensive attention recently in the study of cancer biology. Up to now, several circRNAs have been identified to be closely related to $\mathrm{PCa}$, a clinically heterogeneous and multifactorial disease. For example, circMYLK is overexpressed in PCa tissues and PCa cells [21], and upregulated circ-102004 promotes cell proliferation in PCa [22]. Previously we have also identified two PCarelated circRNAs circ_0057558 and circ_0062019 in PCa [19]. We believe circRNA-based therapy will be a feasible approach for PCa treatment.

In this study, through human circRNA microarray analysis followed by RT-qPCR validation, we identified a circRNA derived from DPP4 gene locus, termed as circDPP4 and found its expression was significantly increased in both clinical PCa tissues and PCa cell lines, implying its oncogenic effect. In vitro gain-of-function and loss-of-function experiments showed that circDPP4 overexpression promoted, whereas circDPP4 knockdown suppressed proliferation, migration and invasion of $\mathrm{PCa}$ cells. Impaired cell proliferation is often caused by cell cycle arrest [23]. Consistently, we found that circDPP4 knockdown suppressed PCa cell cycle progression partly by inhibiting expression of cyclin D1, a well-known oncogene involved in cell cycle progression [24]. Besides, the oncogenic activity of circDPP4 was also verified in nude mouse xenograft model bearing PCa cells.

MiRNAs, a class of small non-coding RNAs, also play a critical role in cancer biology [25]. circRNA could act as ceRNA to regulate gene expression at the post-transcriptional level via sequestering miRNA [26, 27]. This characteristic is similar to that of long non-coding RNAs. Given that circDPP4 is abundant in the cytoplasm, we hypothesized that circDPP4 might serve as a ceRNA to sequester miRNAs in PCa as well. By performing bioinformatics analysis and dual-luciferase reporter assay, we revealed and validated that circDPP4 was able to bind with miR195 , which is widely reported as a tumor suppressor in $\mathrm{PCa}$ and other malignancies $[28,29]$. In agreement with 


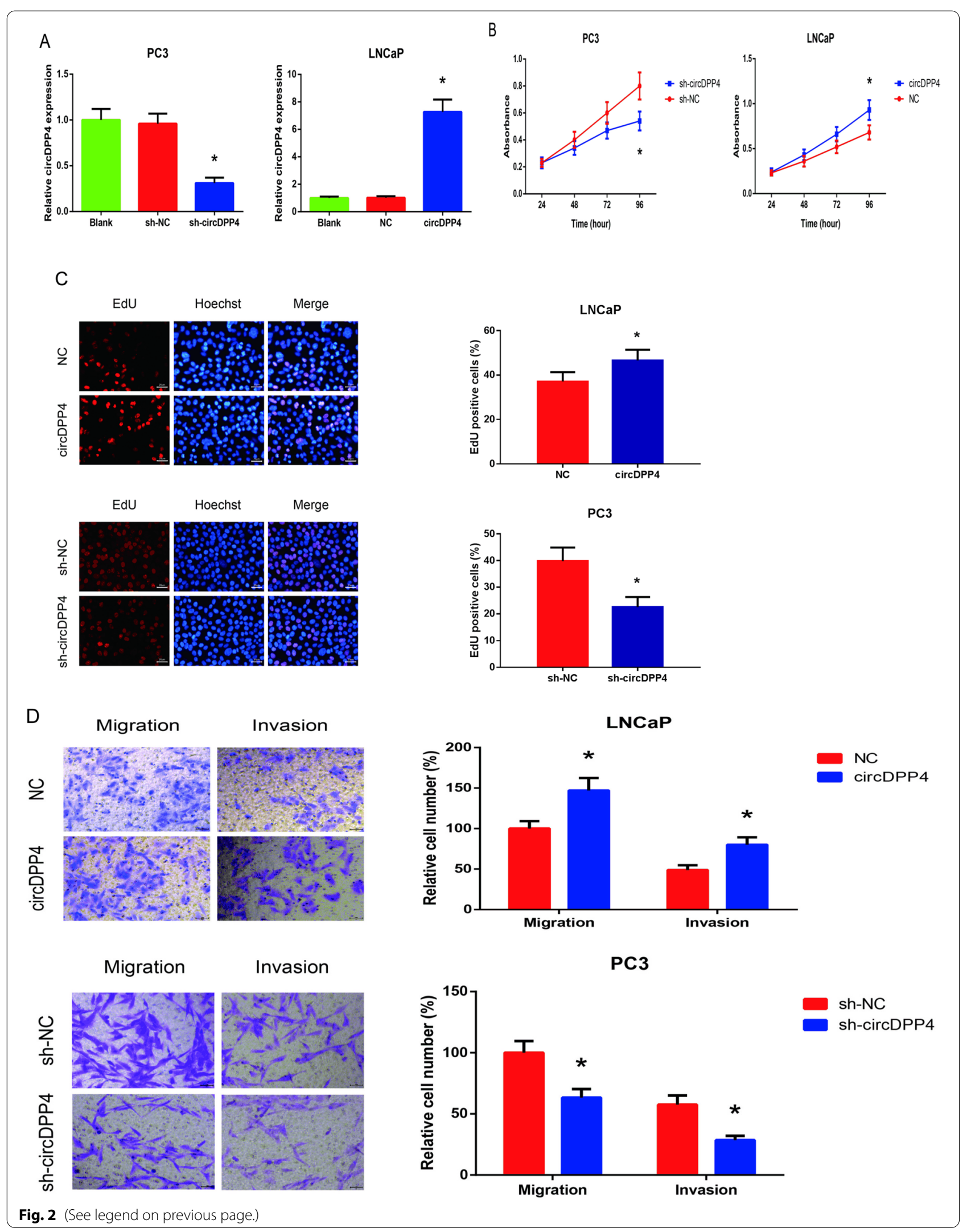



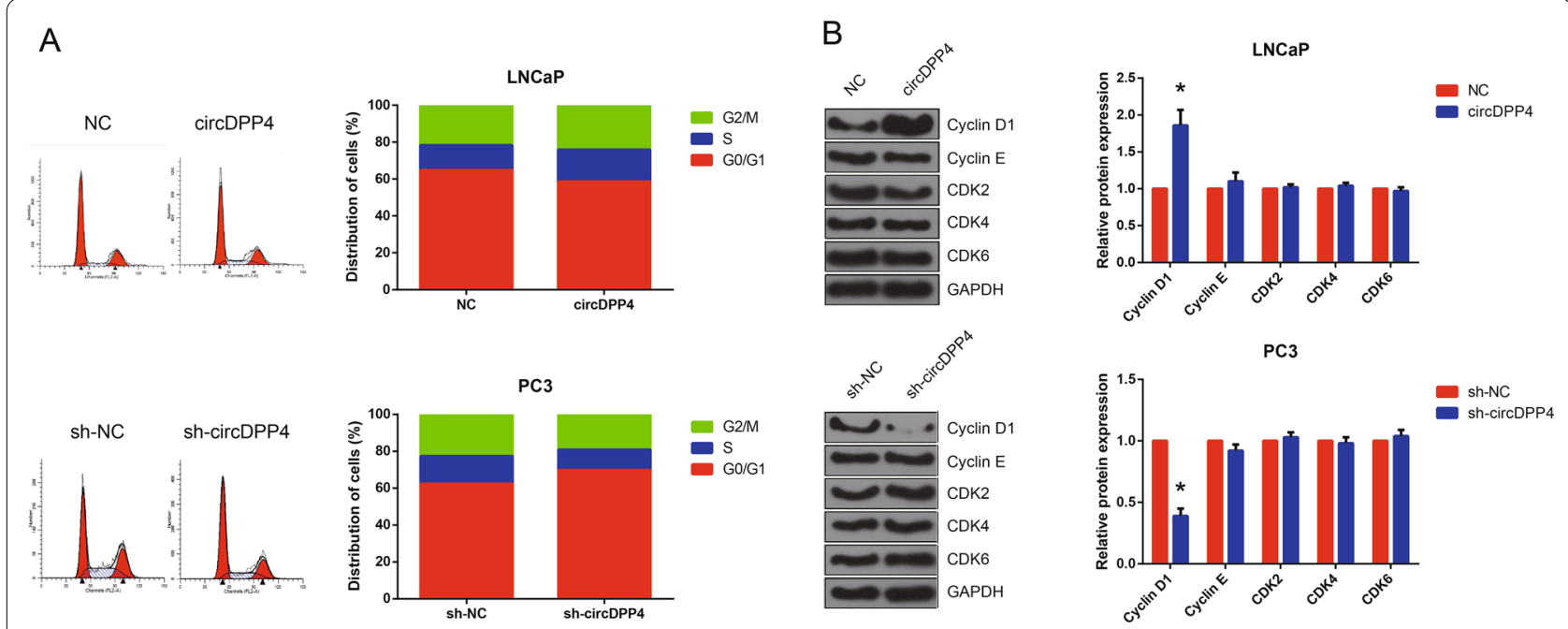

Fig. 3 circDPP4 promotes PCa cell cycle progression. A The cell cycle distribution of PC3 and LNCaP cells after transfection detected by flow cytometric analysis. B Western blot analysis of cell cycle-related protein expression levels in PC3 and LNCaP cells after transfection. ${ }^{*} P<0.05$ vs. sh-NC or empty vector-transfected cells

previous studies, we also showed that miR-195 expression was decreased and negatively correlated with circDPP4 expression in PCa tissues. Besides, through RNA-ISH analysis, we found these two non-coding RNAs co-localized in the cytoplasm of tumor tissues and tumor cell line, implying they are directly correlated.

MiRNAs act as critical signal transduction mediators by negative regulation of their target genes [30], thereby influencing cell fate and function. We then identified Cyclin D1 as a potential direct target of miR-195 in PCa. This interaction has been previously found in osteosarcoma [31] and cervical cancer [32]. Rescue experiments further showed that miR-195 diminished the oncogenic role of circDPP4 in PCa cells, indicating that circDPP4 might function as a ceRNA to sequester and reduce miR195 activity, thus leading to the increase of cyclin D1 expression in PCa.

In conclusion, this is the first report to reveal the expression level and biological function of circDPP4 in PCa. Our results demonstrated that circDPP4 was significantly upregulated in PCa tissues, and circDPP4 promoted cell growth, migration, invasion of $\mathrm{PCa}$ via sponging miR-195 to regulate cyclin D1 expression (Fig. 7). These findings suggested that $\operatorname{circDPP} 4 / \mathrm{miR}$ $195 /$ cyclin D1 axis might involve in the tumorigenesis and progression of $\mathrm{PCa}$ and could become a potential therapeutic target. Further research needs to be conducted to clarify the downstream pathway of circDPP4/ miR-195/cyclin D1 axis.

\section{Abbreviations}

circRNAs: Circular RNAs; PCa: Prostate cancer; ceRNA: Competing endogenous RNA.

\section{Supplementary Information}

The online version contains supplementary material available at https://doi. org/10.1186/s12935-021-02062-z.

Additional file 1: Figure S1. Screening of differentially expressed circRNAs in prostate tissues and matched adjacent normal tissues using microarray analysis. (A) The heatmap; (B) the scatter plot; (C) the volcano plot of differentially expressed circRNAs.

Additional file 2: Figure S2. Cyclin D1 is a downstream target of miR-195 in PCa cells. (A) The predicted miR-195 binding sites in cyclin D1 mRNA $3^{\prime}$-UTR. (B) Dual-luciferase reporter assay performed to validate the direct binding relationship between miR-195 and cyclin D1 mRNA 3'-UTR. (C) Western blot analysis of cyclin D1 protein levels in PC3 and LNCaP cells after transfection. (D) The proliferation of PC3 and LNCaP cells after transfection detected by CCK- 8 assay. ${ }^{*} P<0.05$ vs. mimics control or inhibitor control-transfected cells.

Additional file 3: Figure S3. Co-localization of circDPP4 (green fluorescence) and miR-195 (red fluorescence) in the cytoplasm of PCa cells measured by RNA-fluorescence in situ hybridization (FISH). The nuclei were stained with DAPI (blue fluorescence).

Additional file 4: Table S1. 1021 differentially expressed circRNAs in PCa tissues were identified by microarray analysis.

Additional file 5: Table S2. Correlation between circDPP4 expression and clinicopathological characteristics of PCa patients.

Additional file 6. Representative melting curves and amplification curves of RT-qPCR reaction.

Acknowledgements

Not applicable. 


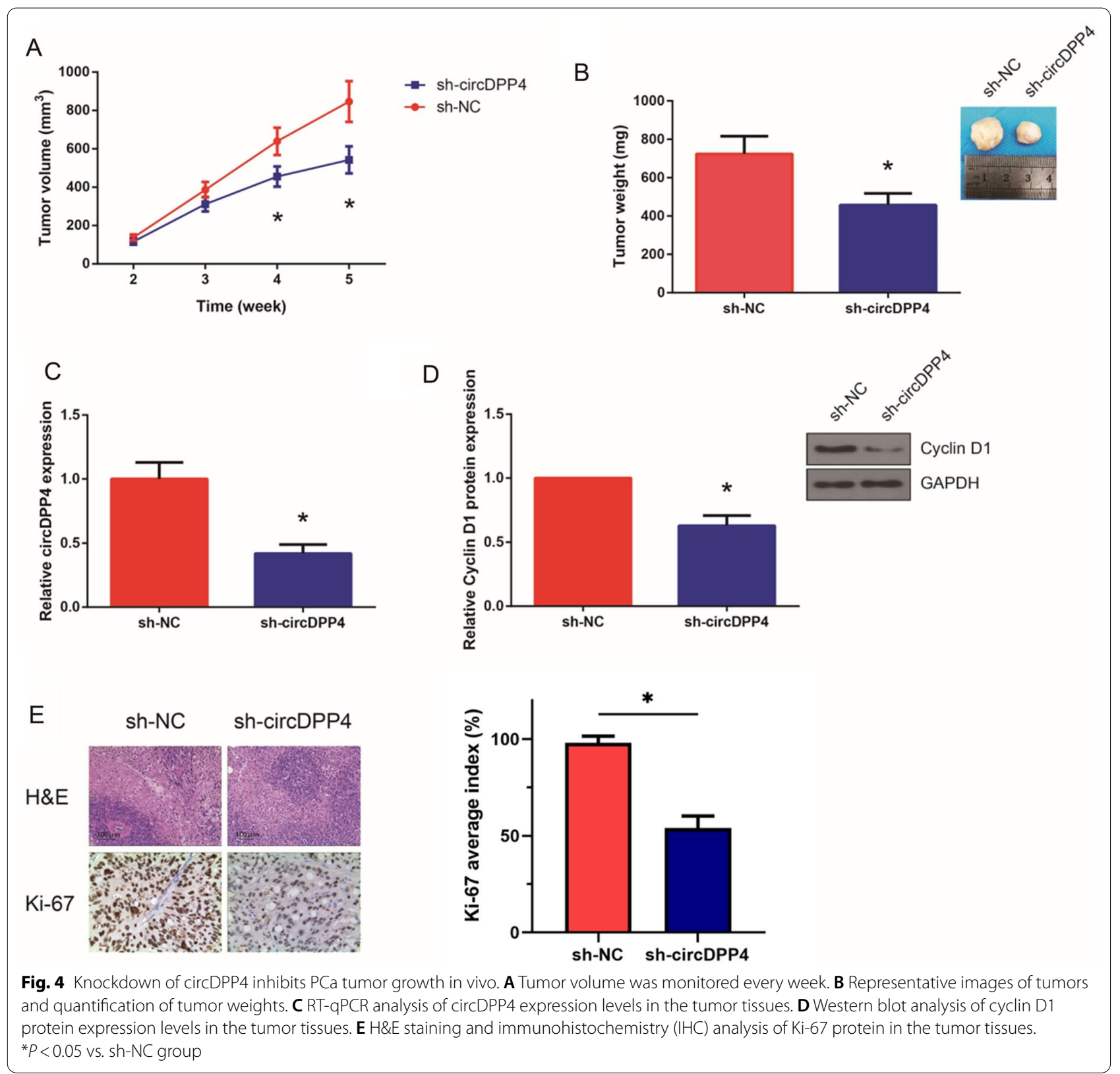

\section{Authors' contributions}

DPY, BY and YJZ participated in the experiment studies, clinical studies, data collection and manuscript writing; QLX, YZ, XZ and JMG interpreted the patient data, and participated in data collection and manuscript writing; TD and $\mathrm{JHZ}$ participated in the project development, data analysis, manuscript editing, and literature research. All contributing authors have agreed to the final version of the manuscript. All authors read and approved the final manuscript.

\section{Funding}

This work was supported by the National Natural Science Foundation of China (No. 81372318), a Grant (No. PWRL2017-07) from Pudong New District

\section{(See figure on next page.)}

Fig. 5 circDPP4 acts as a ceRNA for miR-195 in PCa cells. A Relative expression levels of circDPP4 in the nuclear and cytoplasmic fractions of PCa cells. B The predicted miR-195 binding sites in circDPP4. C Dual-luciferase reporter assay performed to validate the direct binding relationship between circDPP4 and miR-195. ${ }^{*} P<0.05$ vs. mimics control-transfected cells. D The interaction between circDPP4 and miR-195 in PCa cells was measured by RNA-pull down assay. E RT-qPCR analysis of miR-195 expression levels in PC3 and LNCaP cells after transfection. ${ }^{*} P<0.05$ vs. sh-NC or empty vector-transfected cells. F RT-qPCR analysis of miR-195 expression levels in 104 pairs of PCa tissues and adjacent normal tissues. ${ }^{*} P<0.05$ vs. normal tissues. G RT-qPCR analysis of miR-195 expression levels in PCa cell lines and normal counterpart. * $P<0.05$ vs. RWPE1 cells. H The inverse correlation between circDPP4 and miR-195 expression in PCa tissues of 20 randomly selected patients 


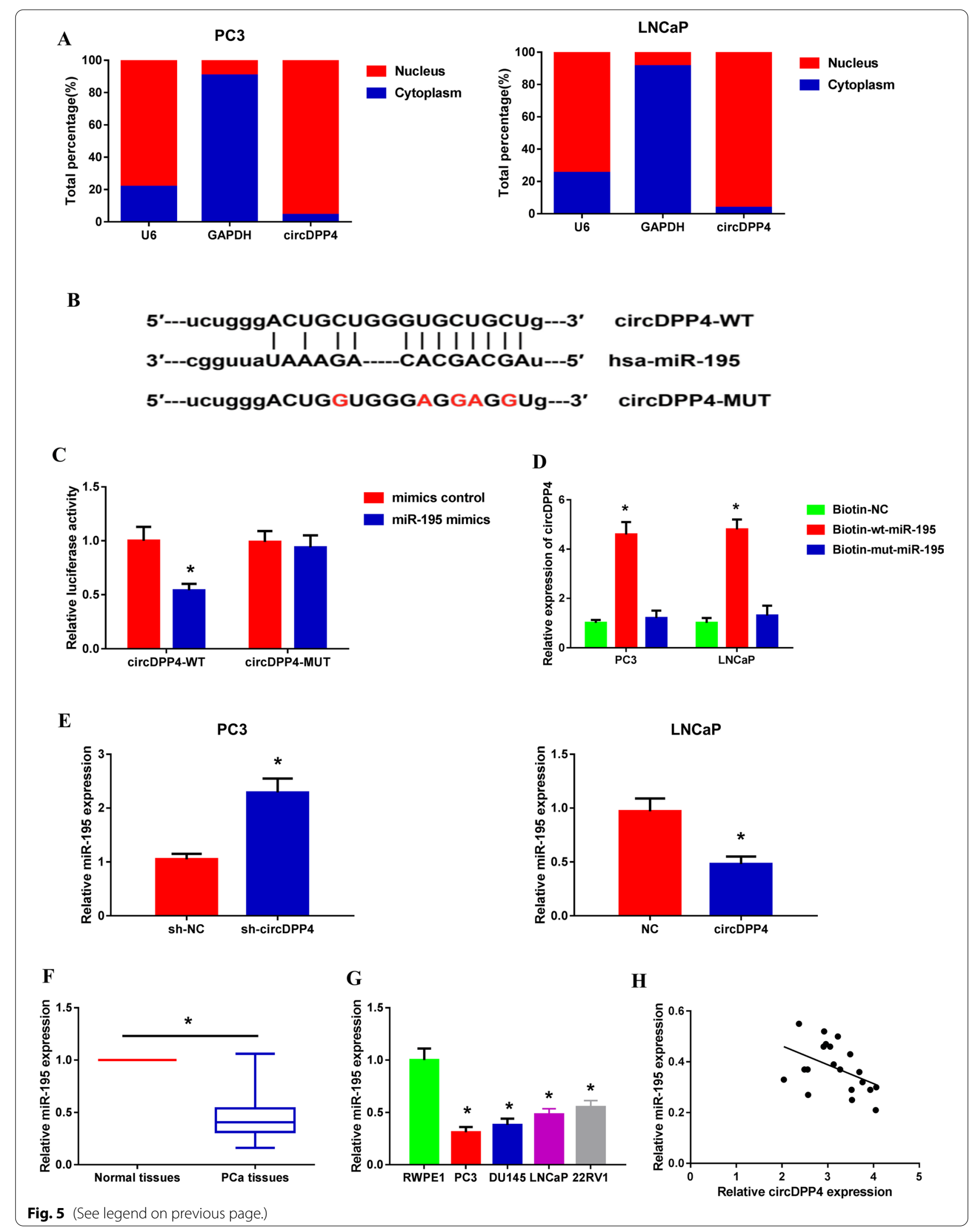



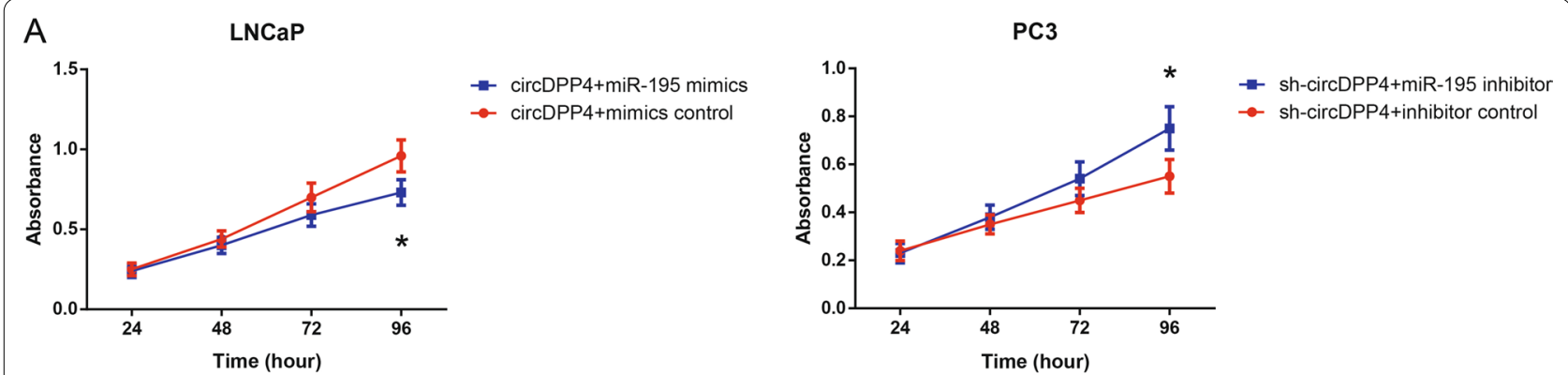

B
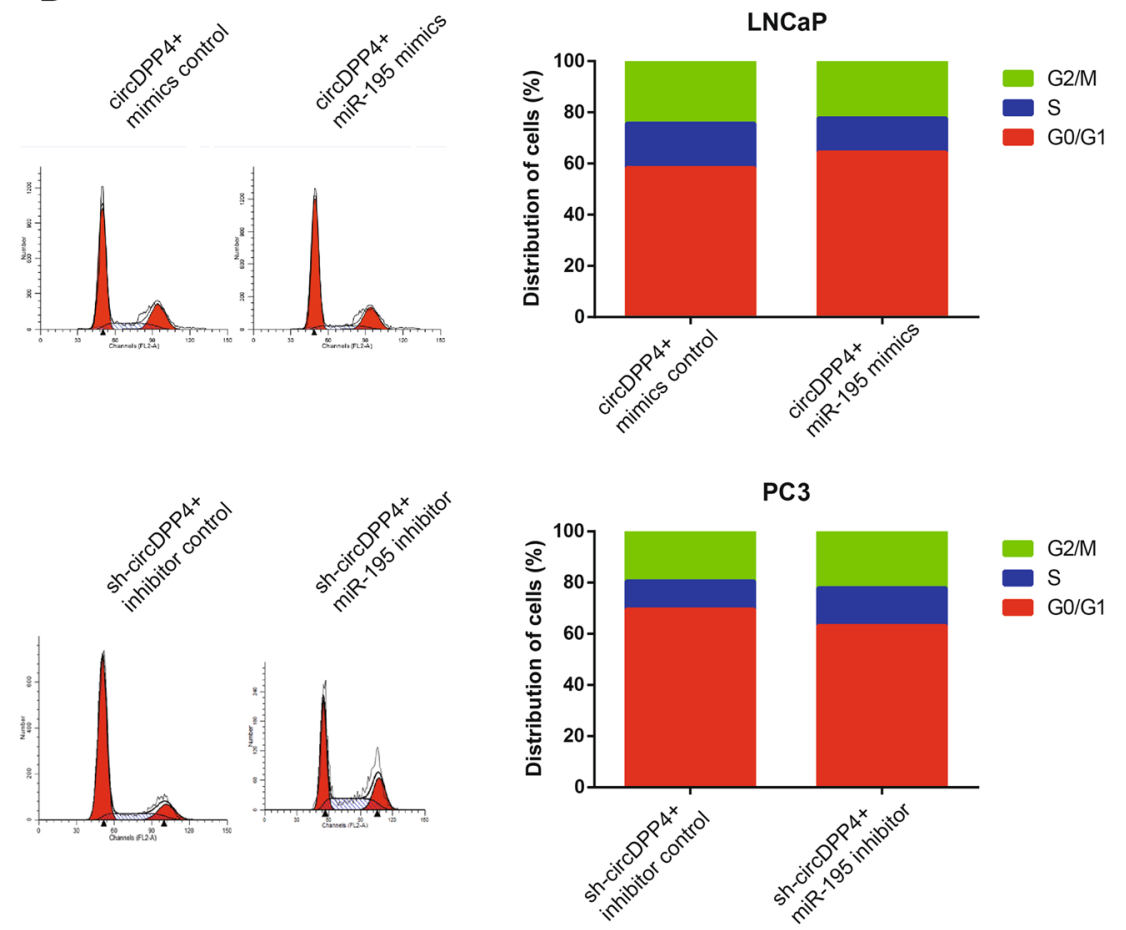

Fig. 6 miR-195 blocks the oncogenic role of circDPP4 in PCa cells. A Proliferation of PC3 and LNCaP cells after transfection detected by CCK-8 assay. B Cell cycle distribution of PC3 and LNCaP cells after transfection detected by flow cytometric analysis. ${ }^{*} P<0.05$ vs. mimics control or inhibitor control-transfected cells

Commission of Health and Family Planning Leading Talent Program (Jianghua Zheng), Shanghai, China and the Science and Technology Commission Fund of Shanghai Fengxian District (No. 20160907), as well as a Grant (No. PW2016D-13) from Pudong New District Commission of Health and Family, Shanghai, China.

\section{Availability of data and materials}

The datasets used and/or analyzed during the current study are available from the corresponding author on reasonable request.

\section{Declarations}

\section{Ethics approval and consent to participate}

The use of human tissues was approved by the Ethics Committee of Shanghai University of Medicine \& Health Sciences Affiliated Zhoupu Hospital, and all patients or their relatives signed the informed consent. All experimental procedures involving the use of animals were approved by the Ethics Committee of Zhongshan Hospital, and all efforts were made to minimize animal suffering, according to the $\mathrm{NIH}$ Guide for the Care and Use of Laboratory Animals.

\section{Consent for publication}

Not applicable.

\section{Competing interests}

The authors declare no competing interests.

\section{Author details}

${ }^{1}$ Department of Laboratory Medicine, Shanghai University of Medicine \& Health Sciences Affiliated Zhoupu Hospital, Shanghai 201318, China.

${ }^{2}$ Department of Urology, Shanghai University of Medicine \& Health Sciences Affiliated Zhoupu Hospital, Shanghai 201318, China. ${ }^{3}$ Department of Urology, Zhongshan Hospital, Fudan University, Shanghai 200032, China. ${ }^{4}$ Department of Laboratory Medicine, The Sixth People's Hospital East Campus, Shanghai Jiao Tong University, Shanghai 201306, China. ${ }^{5}$ Department of Urology, The Sixth People's Hospital South Campus, Shanghai Jiao Tong University, Shanghai 201489, China. 


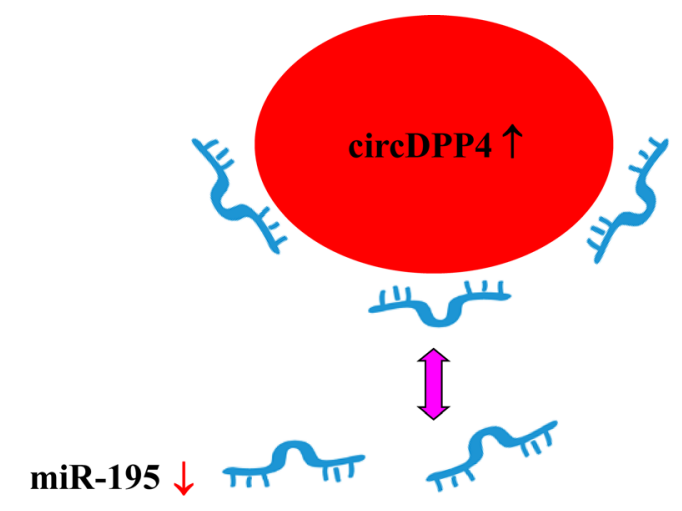

(A negative regulator)
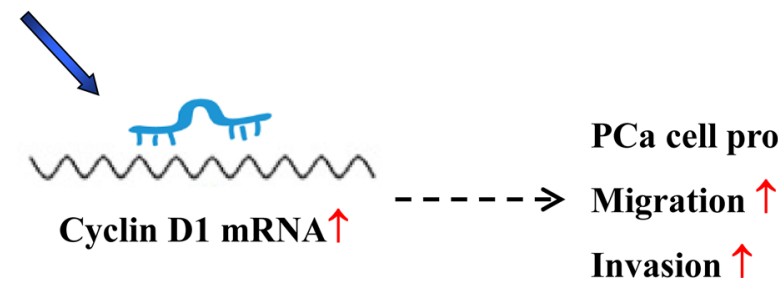

\section{PCa cell proliferation $\uparrow$}

Invasion $\uparrow$

Fig. 7 Schematic of circDPP4/miR-195/cyclin D1 axis in regulating proliferation, migration and invasion of PCa cells

Received: 31 July 2020 Accepted: 28 June 2021

Published online: 16 July 2021

\section{References}

1. Bray F, Ferlay J, Soerjomataram I, Siegel RL, Torre LA, Jemal A. Global cancer statistics 2018: GLOBOCAN estimates of incidence and mortality worldwide for 36 cancers in 185 countries. CA Cancer J Clin. 2018:68(6):394-424.

2. Chen W, Zheng R, Baade PD, Zhang S, Zeng H, Bray F, et al. Cancer statistics in China, 2015. CA Cancer J Clin. 2016:66(2):115-32.

3. Qi D, Wu C, Liu F, Gu K, Shi Z, Lin X, et al. Trends of prostate cancer incidence and mortality in Shanghai, China from 1973 to 2009. Prostate. 2015;75(14):1662-8

4. Qu S, Yang X, Li X, Wang J, Gao Y, Shang R, et al. Circular RNA: a new star of noncoding RNAs. Cancer Lett. 2015;365(2):141-8.

5. Sanger HL, Klotz G, Riesner D, Gross HJ, Kleinschmidt AK. Viroids are single-stranded covalently closed circular RNA molecules existing as highly base-paired rod-like structures. Proc Natl Acad Sci USA. 1976:73(11):3852-6.

6. Chen Y, Li C, Tan C, Liu X. Circular RNAs: a new frontier in the study of human diseases. J Med Genet. 2016:53(6):359-65.

7. Meng S, Zhou H, Feng Z, Xu Z, Tang Y, Li P, et al. CircRNA: functions and properties of a novel potential biomarker for cancer. Mol Cancer. 2017;16(1):94

8. Hansen TB, Jensen TI, Clausen BH, Bramsen JB, Finsen B, Damgaard CK, et al. Natural RNA circles function as efficient microRNA sponges. Nature. 2013;495(7441):384-8.

9. Ashwal-Fluss R, Meyer M, Pamudurthy NR, Ivanov A, Bartok O, Hanan M, et al. CircRNA biogenesis competes with pre-mRNA splicing. Mol Cell. 2014;56:55-66.

10. Xiong X, Feng J, Yang X, Li H, Shi Q, Tao J, et al. Circular RNA CDR1as promotes tumor progression by regulating miR-432-5p/E2F3 axis in pancreatic cancer. Cancer Cell Int. 2021;21:1-10.

11. Tao L-J, Pan X-Y, Wang J-W, Zhang L, Tao L-S, Liang C-Z. Circular RNA circANKS1B acts as a sponge for miR-152-3p and promotes prostate cancer progression by upregulating TGF-a expression. Prostate. $2021 ; 81: 271-8$
12. Montalto FI, De Amicis F. Cyclin D1 in cancer: a molecular connection for cell cycle control, adhesion and invasion in tumor and stroma. Cells. 2020;9(12):2648.

13. Cao Z, Shi $X$, Tian F, Fang $Y$, Wu B, Mrdenovic S, et al. KDM6B is an androgen regulated gene and plays oncogenic roles by demethylating H3K27me3 at cyclin D1 promoter in prostate cancer. Cell Death Dis. 2021;12:2.

14. Shi Q, Li Y, Li S, Jin L, Lai H, Wu Y, et al. LncRNA DILA1 inhibits cyclin D1 degradation and contributes to tamoxifen resistance in breast cancer. Nat Commun. 2020;11:5513.

15. Luangdilok S, Wanchaijiraboon $P$, Chantranuwatana $P$, Teerapakpinyo C, Shuangshoti S, Sriuranpong V. Cyclin D1 expression as a potential prognostic factor in advanced KRAS-mutant non-small cell lung cancer. Transl Lung Cancer Res. 2019;8(6):959-66.

16. Wu SY, Lan SH, Wu SR, Chiu YC, Lin XZ, Su IJ, et al. Hepatocellular carcinoma-related cyclin D1 is selectively regulated by autophagy degradation system. Hepatology. 2018;68(1):141-54.

17. Duan X, Shen N, Chen J, Wang J, Zhu Q, Zhai Z. Circular RNA MYLK serves as an oncogene to promote cancer progression via microRNA-195/cyclin D1 axis in laryngeal squamous cell carcinoma. Biosci Rep. 2019:39(9):BSR20190227.

18. Wu Z. MiR-195 connects IncRNA RUNX1-IT1 and cyclin D1 to regulate the proliferation of glioblastoma cells. Int J Neurosci. 2021. https://doi. org/10.1080/00207454.2021.1881090.

19. Xia Q, Ding T, Zhang G, Li Z, Zeng L, Zhu Y, et al. Circular RNA expression profiling identifies prostate cancer-specific circRNAs in prostate cancer. Cell Physiol Biochem. 2018;50(5):1903-15

20. Livak KJ, Schmittgen TD. Analysis of relative gene expression data using real-time quantitative PCR and the 2(-Delta Delta C(T)) method. Methods. 2001;25(4):402-8.

21. Dai Y, Li D, Chen X, Tan X, Gu J, Chen M, et al. Circular RNA myosin light chain kinase (MYLK) promotes prostate cancer progression through modulating Mir-29a expression. Med Sci Monit. 2018;24:3462-71.

22. Si-Tu J, Cai Y, Feng T, Yang D, Yuan S, Yang $X$, et al. Upregulated circular RNA circ-102004 that promotes cell proliferation in prostate cancer. Int J Biol Macromol. 2018;22:1235-43.

23. Hanahan D, Weinberg RA. Hallmarks of cancer: the next generation. Cell. 2011;144(5):646-74.

24. Diehl JA. Cycling to cancer with cyclin D1. Cancer Biol Ther. 2002;1(3):226-31 
25. Negrini M, Ferracin M, Sabbioni S, Croce CM. MicroRNAs in human cancer: from research to therapy. J Cell Sci. 2007;120(Pt 11):1833-40.

26. Karreth FA, Pandolfi PP. ceRNA cross-talk in cancer: when ce-bling rivalries go awry. Cancer Discov. 2013;3(10):1113-21.

27. Yan X, Hu Z, Feng Y, Hu X, Yuan J, Zhao SD, et al. Comprehensive genomic characterization of long non-coding rnas across human cancers. Cancer Cell. 2015;28(4):529-40.

28. Cai C, Chen QB, Han ZD, Zhang YQ, He HC, Chen JH, et al. miR-195 inhibits tumor progression by targeting RPS6KB1 in human prostate cancer. Clin Cancer Res. 2015;21(21):4922-34.

29. Wu J, Ji A, Wang X, Zhu Y, Yu Y, Lin Y, et al. MicroRNA-195-5p, a new regulator of Fra-1, suppresses the migration and invasion of prostate cancer cells. J Transl Med. 2015;13:289.
30. Bartel DP. MicroRNAs: target recognition and regulatory functions. Cell. 2009;136(2):215-33.

31. Han K, Chen X, Bian N, Ma B, Yang T, Cai C, et al. MicroRNA profiling identifies MiR-195 suppresses osteosarcoma cell metastasis by targeting CCND1. Oncotarget. 2015;6(11):8875-89.

32. Li Z, Wang H, Wang Z, Cai H. MiR-195 inhibits the proliferation of human cervical cancer cells by directly targeting cyclin D1. Tumour Biol. 2016;37(5):6457-63.

\section{Publisher's Note}

Springer Nature remains neutral with regard to jurisdictional claims in published maps and institutional affiliations.
Ready to submit your research? Choose BMC and benefit from:

- fast, convenient online submission

- thorough peer review by experienced researchers in your field

- rapid publication on acceptance

- support for research data, including large and complex data types

- gold Open Access which fosters wider collaboration and increased citations

- maximum visibility for your research: over $100 \mathrm{M}$ website views per year

At BMC, research is always in progress.

Learn more biomedcentral.com/submissions 